\title{
Long term in-vivo studies of a photo-oxidized bovine osteochondral transplant in sheep Margarete K Akens $^{* 1}$, Brigitte von Rechenberg${ }^{1}$, Pedro Bittmann², Daniel Nadler², Kati Zlinszky1 and Jörg A Auer ${ }^{1}$
}

Address: ${ }^{1}$ Musculoskeletal Research Unit, Department of Veterinary Surgery, University of Zurich, 8057 Zurich, Switzerland and 2Sulzer Orthopedics Ltd, 8401 Winterthur, Switzerland

E-mail: Margarete K Akens* - makens@vetklinik.unizh.ch; Brigitte von Rechenberg - bvrech@vetchir.unizh.ch;

Pedro Bittmann - Peter.Bittmann@sulzer.com; Daniel Nadler - daniel.nadler@sulzer.com; Kati Zlinszky - zlin@vetpath.unizh.ch; Jörg A Auer - jauer@vetchir.unizh.ch

${ }^{*}$ Corresponding author

BMC Musculoskeletal Disorders 200I, 2:9

Accepted: 30 November 2001

This article is available from: http://www.biomedcentral.com/I47I-2474/2/9

(c) 200 I Akens et al; licensee BioMed Central Ltd. Verbatim copying and redistribution of this article are permitted in any medium for any non-commercial purpose, provided this notice is preserved along with the article's original URL. For commercial use, contact info@biomedcentral.com

\begin{abstract}
Background: Articular cartilage has limited capacity to repair. Defects greater than $3 \mathrm{~mm}$ heal with formation of inferior fibrous cartilage. Therefore, many attempts have been made to find the ideal graft for larger cartilage lesions. Different grafts, such as untreated or cryopreserved osteochondral transplants, have been used with variable success.

Methods: Photo-oxidized osteochondral grafts were implanted in both femoral condyles of one ovine knee. Untreated xenogeneic and autogeneic grafts served as controls. Three groups of 8 sheep each were formed and they were sacrificed 6,12 or 18 months after surgery.

Results: The macroscopic evaluation of the condyle and graft showed a well-maintained cartilage surface in most grafts at all time points. However, the host cartilage matrix deteriorated considerably in all xenogeneic, most autogeneic and fewer of the photo-oxidized grafts at 12 and 18 months, respectively. The blue colour of the photo-oxidized grafts resulting from the process of photo-oxidation was visible in all grafts at 6 months, had diminished at 12 months and had completely disappeared at 18 months after surgery. Histologically a loss of matrix staining was almost never noticed in untreated xenografts, transiently at 6 months in photo-oxidized grafts and increased at 12 and 18 months. Fusion between graft and host cartilage could be seen in photooxidized grafts at 12 and 18 months, but was never seen in autografts and xenografts.

Conclusions: The photo-oxidation of osteochondral grafts and its use as transplant appears to have a beneficial effect on cartilage and bone remodelling. Osteochondral grafts pre-treated with photo-oxidation may be considered for articular cartilage replacement and therefore may delay artificial joint replacements in human patients.
\end{abstract}

\section{Background}

Destruction of articular cartilage due to acute trauma or degenerative joint disease (DJD) results in most instanc- es in repair with inferior cartilage tissue [1-3]. The repair mechanisms depend on several parameters: 1 . the size of the original lesion; 2 . the overall status of the articular 
cartilage; 3. the condition of the synovial membrane; and 4. the successful removal of the causative factors, such as mechanical overload, loose bone fragments between the opposing articular surfaces, or instability of the joint due to ligament damage $[4,5]$.

Hyaline cartilage lesions smaller than $3 \mathrm{~mm}$ of size in diameter repair with normal hyaline cartilage, whereas lesions greater than $3 \mathrm{~mm}$ are initially replaced by fibrous cartilage that eventually degenerates into fibrous tissue $[4,6]$. This inferior repair tissue is not capable of withstanding the mechanical loads exerted on the tissue during motion and the result is eburnation of the subchondral bone [7]. Clinical symptoms of joint disease in human and animal patients include joint distension, decreased range of motion, and pain during weight bearing associated with lameness. In severe cases the pain may be excruciating. Joint replacement with artificial joint prostheses is an option for several joints in human patients and in some instances also in small animals. In young human patients, however, a better option than metal implants is required, because the clinical problem of aseptic loosening and wearing out of joint prostheses have not yet been solved.

Therefore, grafting procedures to replace damaged cartilage with biological tissue is considered an option to delay the implantation of artificial joints [8-12].

Different grafts, osteochondral - [13-15], periosteal $[16,17]$, and chondral and chondrocytes in various carrier systems $[18,19]$, have been used, but the results are questionable [20]. Although initially they showed some promise, problems with matrix degradation of the grafts, graft dislocation and replacement with inferior tissue prevented their successful clinical application [20]. Nevertheless, to date, osteochondral -, fresh - and cryopreserved grafts are used in man and animals, either as a single transplant [21-24] or as multiple transplants [25]. Fresh autografts proved to be superior to fresh or cryopreserved allografts $[12,23,26,27]$. Cryopreservation reduces the viability of chondrocytes within the grafts, which results in inferior remodelling activity of the subchondral bone compared to autografts $[13,23,26,27]$.

Apart from graft survival, another crucial parameter of transplantation is the transition between graft and host tissue. The gap between transplant and host tissue was held responsible for the initiation of cartilage degeneration, possibly as a result of micromotion between transplant and host tissue $[8,20,28]$. Additionally, penetration of synovial fluid into the host-graft interface results in resorption within the subchondral bone area and cyst formation [29]. A good surgical technique that results in a tight fit between the host and graft tissue is, therefore, essential [11,20,30].

The search for the ideal graft for articular cartilage replacement is continuing. The reduced ability of articular cartilage to regenerate and integrate an osteochondral transplant, and the subsequent degradation of the transplanted matrix are problems that remain to be solved [20].

The use of an osteochondral xenograft, pre-treated with photo-oxidation was investigated in an experimental sheep model. Photo-oxidation involves the immersion of the grafts in methylene blue solution and exposure to light. This process affects the sulphur-containing and aromatic amino acids of the collagen fibrils. An increase of oxygen uptake and $\mathrm{CO}_{2}$ evolution are due to the cyclic nucleus of these amino acids and leads to a rupture of the ring system. In studies with cardiac valve replacement, photo-oxidation was shown to reduce antigenicity of porcine pericardial tissue [31,32]. The photo-oxidation procedure has not previously been applied to bone and articular cartilage. Previous in-vitro studies with the photo-oxidized cartilage showed that this process leaves a transplant with a mechanically stronger matrix devoid of living chondrocytes [33]. In addition, it was demonstrated that the incubation of photo-oxidized cartilage in combination with native, untreated cartilage resulted in decreased release of local mediators, such as nitric oxide (NO) and prostaglandin $\mathrm{E}_{2}\left(\mathrm{PGE}_{2}\right)$, and reduced activity of neutral metalloproteinases that are known to be involved in cartilage degeneration [34,35].

Using the press-fit technique [11], photo-oxidized osteochondral transplants were implanted into the femoral condyles of sheep to evaluate the feasibility of in-vivo transplantation. Short and long term survival of the grafts and the adjacent host matrix were followed macroand microscopically at six, twelve and eighteen months.

\section{Methods}

\section{Preparation of photo-oxidized grafts}

Bovine shoulder joints from eighteen-month old cattle containing macroscopically normal articular cartilage were collected twelve hours after slaughter. Cartilage was considered normal if it was shining bluish - white with no discoloration, wear lines, or other signs of degradation present. The osteochondral transplants were harvested under aseptic conditions. All soft tissues were removed and remnants of synovial fluid flushed away with sterile physiological saline (0.9\% w/v). Using a diamond-coated hollow drill bit (Draenert, Munich, Germany) with built-in irrigation, standardised osteochondral transplants (5.5 mm in diameter; $6 \mathrm{~mm}$ in length) were harvested from the articular surface of the humeral head 
and immediately transferred into sterile vials filled with physiologic saline. After extensive rinsing, the transplants were placed in a 0.01 percent methylene blue solution in phosphate buffered saline (PBS) (Life Technologies AG, Basel, Switzerland) for $12 \mathrm{~h}$ to allow dye penetration. The transplants were then transferred into a customised device and photo-oxidized under aseptic conditions by exposure to light under controlled conditions (light intensity, temperature and exposition time (patent pending)). After completion of the photo-oxidation process the transplants were transferred to a second closed and sterile circuit where the excess methylene blue dye was washed off with PBS. The photo-oxidized osteochondral transplants were stored in sterile ethanol $(50 \% \mathrm{v} / \mathrm{v})$ at $4^{\circ} \mathrm{C}$. The maximal storage time before transplantation was two weeks.

Control xenogeneic, untreated osteochondral grafts were harvested immediately prior to transplantation from fresh bovine shoulder joints under aseptic conditions, washed and kept in saline for only a few minutes before use. Care was taken to assure short storage time in saline solution to avoid osmotic swelling of the cartilage. Even harvesting grafts from a different joint, congruency was not a major problem because of the size of the graft ( 5.5 $\mathrm{mm}$ in diameter). Problems with joint congruency were mainly related to surgical procedure when the graft was not placed perpendicular to the joint surface. Attention was paid to the cartilage thickness of the graft, but differences between graft and host cartilage could not always be avoided.

The autogeneic ovine graft was harvested from the weight bearing area of the femoral condyle, washed in PBS and transplanted either from the medial to the lateral condyle or vice versa in the same joint. A photo-oxidized graft was used to fill the defect resulting at the autograft-harvesting site.

\section{Experimental animals}

Twenty-four adult female Swiss crossbred sheep (dentes incisivi had completely changed) were randomly allocated to six-, twelve-, and eighteen-month survival groups (eight animals per group). Grafts that were placed in the lateral and medial femoral condyles of one stifle joint in the weight bearing area. The total number of grafts per group was sixteen, ten were photo-oxidized, two autogeneic and four untreated xenogeneic. In a preliminary study, the results at two months after implantation with auto- and xenografts were poor, and therefore only two condyles were transplanted with autogeneic and four condyles with untreated xenogeneic grafts in each group (six, twelve and eighteen months). Since experimental animals were used and the main focus of this time course study was placed on the photo-oxidized grafts, the use of fewer control sheep was justified for ethical reasons. Furthermore, it was not expected that the results with the control transplants would improve with longer experimental times.

An animal utilisation study committee has approved the study and the sheep were kept in compliance with the law of animal welfare (Permission number: 145/97) Two weeks prior to surgery the sheep were examined clinically, dewormed and accustomed to their new environment. Prior to induction of anaesthesia, food was withheld for thirty-six hours and the hind limbs of the sheep were clipped.

\section{Anaesthesia and surgical procedure}

The animals were sedated using xylazine intramuscularly (0.02 mg/kg; Rompun ${ }^{\circledR}$, Bayer AG, Germany) and anaesthesia was induced with ketamine intravenously (4 $\mathrm{mg} / \mathrm{kg}$; Narketan ${ }^{\circledR}$, Chassot AG, Switzerland). Anaesthesia was maintained with isoflurane (Forene ${ }^{\circledR}$, Abbott AG, Baar, Switzerland) in oxygen. The sheep were positioned in laterodorsal recumbency with the hyperflexed hindlimb fixed to the table in upright position. A long diagonal skin incision in the shape of a stretched $S$ was made over the knee joint. The incision was started proximally medial to the patella and ended laterally, $2 \mathrm{~cm}$ distal to the tibia plateau. Subsequently, an arthrotomy was performed into the medial and lateral joint compartment, parallel to the straight patellar ligament. A partial resection of the intra-articular fat pad facilitated the exposure of the weight-bearing surface of both condyles where the host bed for the transplant was created with a specially designed drill bit conforming to the prepared grafts. The drill bit had an outer diameter of $5.4 \mathrm{~mm}, 0.1$ $\mathrm{mm}$ less than the outer diameter of the photo-oxidized and xenografts. In addition there was a stop mechanism, so that the depth $(6 \mathrm{~mm})$ drilled was exactly the same as that of the graft, attached, allowing an exact fit of the graft (pressfit technique). Small variation in placing the graft within the weightbearing area could not be avoided. The autograft was harvested with a drill bit, which had the outer diameter of the photo-oxidized graft. The diameter of the autograft was smaller because of the tissue loss due to drill hollow bit kerf (Draenert, Munich, Germany). Therefore the hole for the autograft was $4.4 \mathrm{~mm}$ ). The size of the graft was chosen to achieve congruency of the joint surface. The convexity of the ovine femoral is greater compared to the human femoral condyle.

Arthrotomy closure was routine using 2-O polyglycolic acid suture material (Vicryl, Ethicon, Norderstedt, Germany) for adaptation of the joint capsule, the fascia and subcutaneous tissue. Each layer was closed in a simple continuous pattern. Surgical staples (Signet $35 \mathrm{~W}^{\circledR}$, Auto Suture, Connecticut, USA) were used to close the skin. 
Additionally, a gauze sponge was stapled over the incision. Postoperative antibiotics (penicillin G (30.00o IU/ $\mathrm{kg}$, Hoechst AG, Germany) and gentamicin $(7 \mathrm{mg} / \mathrm{kg}$, Streuli \& Co. AG, Switzerland) were administered prophylactically twice a day and nonsteroidal analgesic (flunixin meglumine, $1.1 \mathrm{mg} / \mathrm{kg}$, Essex Pharma GmbH, Germany) once a day for three days.

After surgery the animals were confined in groups in a small stable for the first 5 days and allowed normal weight bearing thereafter in a larger indoor pen for six weeks. For the rest of the experimental period they were allowed to roam free on a pasture. After six-, twelve-, and eighteen-months respectively a group of eight sheep each were sacrificed. The stifle joints were immediately harvested for further evaluation.

\section{Macroscopic evaluation}

The joints were dissected carefully and the appearance of the articular cartilage assessed immediately. Emphasis was placed on 1) graft position 2) graft surface 3) grafthost interface and 4) adjacent host cartilage. Additionally osteophyte development, synovial membrane pathology and degree of joint effusion were recorded. The position of the graft was considered normal if no obvious dislocation was present and if the graft surface was at the same level as the adjacent host cartilage. The surface of the graft was considered normal if no clefts, no dull, blood tinged, discoloration with a cobblestone-like appearance of the articular cartilage was present. In case of the autogeneic or xenogeneic grafts a shiny, whitish colour was judged normal. In the pre-treated, photo-oxidized grafts the intensity of the bluish colour was recorded. The host-graft interface was evaluated closely. Special emphasis was placed on whether clefts were present, or whether a cartilage-like bond had formed between the graft and the host cartilage. The host cartilage was checked for signs of early degeneration, such as change of colour, fibrillation, cleft formation or flaking cartilage. Again, the host cartilage was considered normal if the surface was glossy and bluish-white, and no signs of matrix degradation were noticed. Each joint was recorded photographically.

\section{Microradiographs}

Blocks $1.5 \times 1.5 \mathrm{~cm}$ containing the graft with the adjacent cartilage and bone were cut with of a bone saw (Biro, Modell 3334, Matti AG, Lenzburg, Switzerland). Microradiographs (Faxitron Series - Cabinet X-ray System, Hewlett Packard, Oregon, USA) of the blocks were taken to assess the trabecular bone structure.

\section{Histology}

The bone samples were then fixed in 4 percent buffered formaldehyde for at least one week at $4^{\circ} \mathrm{C}$, followed by washing and dehydration with graded ethanols and clearing with xylene and embedding in methylmethacrylate (HistoDur ${ }^{\circledR}$, Leica, Switzerland) as described elsewhere [36]. Sections $200 \mu \mathrm{m}$ thick were cut from the polymerised blocks, using a sawing microtome (Sawing microtome Leica SP 1600) and mounted on acrylglas slides (Perspex GS Acrylglas PMMA/Opal 1013, Wachendorf AG, Basel, Switzerland). The sections were ground with a grinding machine (Planopol-V, Struers, Rødovre, Denmark), to an appropriate thickness of 30$40 \mu \mathrm{m}$. The sections were subsequently polished with polishing cloths (DP-MOL, DP-NAP with adhesive back), containing polycrystalline diamonds (Diamantpaste $\mathrm{P}^{\circledR}(3 \mu \mathrm{M}$ and $1 \mu \mathrm{M}$ grain size) and lubricants (DPLubricant Blue and $\left.\operatorname{Red}^{\circledR}\right)$. The sections were subsequently surface stained with toluidine blue for histological evaluation. One of the ten photo-oxidized transplants from the eighteen-month group was used for a viability test and could not be evaluated histologically.

\section{Evaluation of sections}

Qualitative, blind evaluation of the histological sections was carried out using the scoring system listed in table 1 . The assessment was based on the appearance of 1) the cartilage, 2) the mineralised cartilage, 3) the subchondral bone and 4) the general appearance of the graft and the surrounding tissue. Each category was subdivided in further parameters (Table 1). The appearance of the graft included its overall position and integrity relative to the surrounding tissues, whereas its bonding to the host tissue was judged by the presence or absence of a cleft between the graft and the adjacent cartilage matrix. Matrix staining of articular cartilage, cell morphology and density, and the integrity of the tidemark were evaluated for the cartilaginous regions. For the general appearance (four above) new bone formation, remodelling of the old subchondral bone and the interface between graft and host bone matrix were assessed.

Scores were given ranging from o to 2, with o indicating the lowest and 2 the best results (Table 1). Two independent scientists in blinded fashion evaluated the sections and the scores were averaged. Each parameter was evaluated in graft and host except the border of the graft. The average mean of all scores for each group and time point is shown in figure 1.

Statistical analysis of scores was performed using the Kruskal - Wallis - test followed by Dunns post test only for photo-oxidized grafts at the different time points: six, twelve and eighteen months; because of the low numbers of xenografts and autografts in the long term survival groups. A p value $<0.05$ was considered to be significant. 


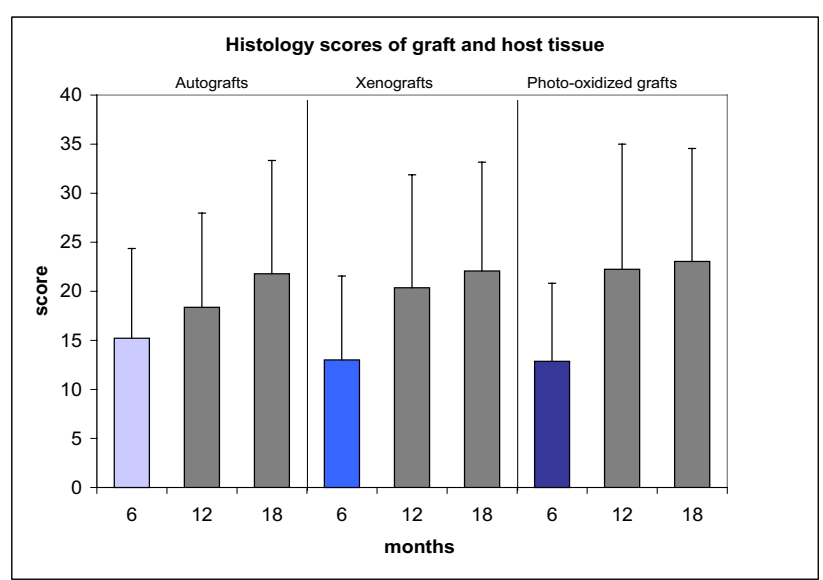

Figure I

Average of histology scores of all categories were calculated and means for each group and time points are shown.

\section{Results}

\section{Clinical evaluation}

The sheep showed a slight lameness on the operated limb for eight to ten days after surgery. Furthermore, the joint swelling evident in six sheep regressed within two weeks after surgery. All except one of the sheep walked without lameness when turned out to pasture. In this sheep, the collateral stifle ligament was accidentally severed during the surgical approach. The ligament was reconstructed using resorbable suture material (Vicryl, Ethicon, Norderstedt, Germany) and this sheep was kept inside until sacrifice. One sheep from the eighteen-month; xenogeneic osteochondral graft group acquired a clostridium enterotoxaemia infection fourteen months after surgery and died. No other complications occurred.

\section{Macroscopic evaluation of cartilage surface}

The joints of all groups appeared normal except in two sheep (six month untreated xenografts) where joint effusion and slight synovitis was found. One effusion was fresh and of unknown origin, whereas the second was caused by joint instability because of the severed collateral stifle ligament (see above). The synovial membrane of the other sheep showed no signs of chronic or acute inflammation macroscopically.

\section{6 - months group}

At six months all photo-oxidized grafts $(\mathrm{n}=10)$ were still blue in colour, although less intensive (Figure 2). The blue colour results from the process of photo-oxidation, because the methylene blue solution cannot be removed completely. Four of ten transplants collapsed slightly into the defect, whereas six stayed in place. The interface between graft and host cartilage was still visible and a slight discoloration and loss of glossy appearance was

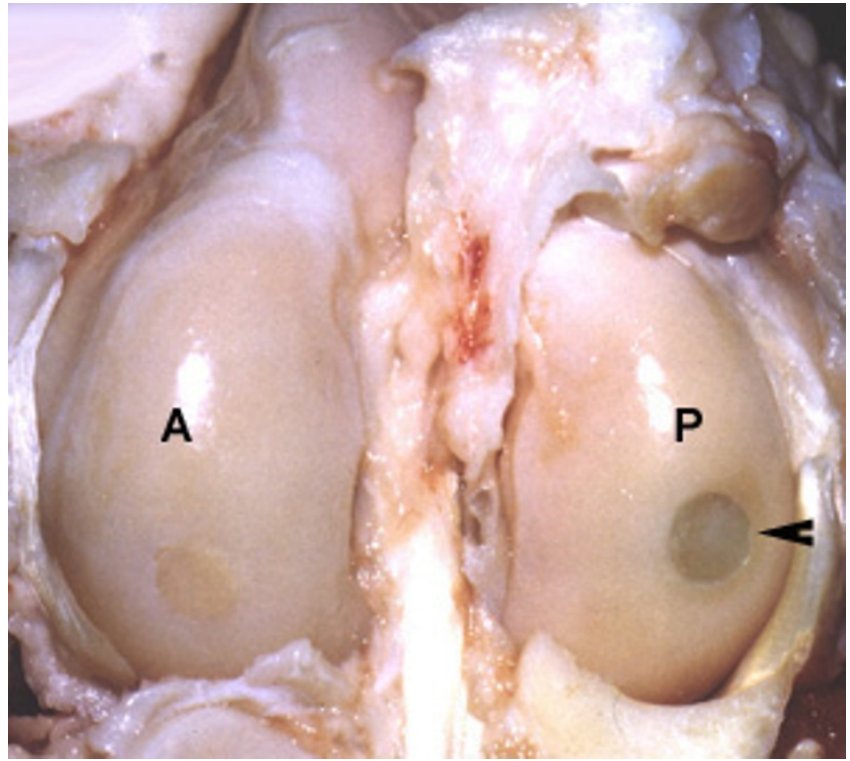

\section{Figure 2}

Macroscopic appearance of femoral condyles: autogeneic transplant $(A)$ at the medial condyle and a photo-oxidized osteochondral transplants $(P)$ at the lateral condyle after 6 months (<: small area of dull cartilage).

noted in the adjacent host cartilage in four cases. The autogeneic osteochondral transplants $(n=2)$ stayed in place and the graft-host interface was still visible. One graft showed a rough surface. Three of the xenogeneic osteochondral transplants $(n=4)$ had collapsed 1 to 2 $\mathrm{mm}$ into the defect.

\section{2 - months group}

In nine of the photo-oxidized osteochondral grafts $(\mathrm{n}=$ 10) the blue colour had disappeared (Figure 3). The surface of cartilage on the grafts appeared white, slightly uneven and, in seven cases shiny. Only the graft that still showed a bluish colour was surrounded by 1 to $2 \mathrm{~mm}$ of dull host cartilage and the host-graft interface was still visible. Seven photo-oxidized osteochondral transplants showed evidence of a union between the graft cartilage and the adjacent host cartilage. The autogeneic osteochondral transplants $(n=2)$ stayed in place, showed a slightly uneven surface and the graft-host interface was still visible. Half of the xenogeneic transplants $(n=4)$ stayed in place and were surrounded by 1 to $2 \mathrm{~mm}$ of dull host cartilage. The other half of the transplants had sunk and had a very rough surface (Figure 4 ).

\section{8 - months group}

All photo-oxidized osteochondral transplants $(n=10)$ appeared white. Six transplants showed evidence for a union between the graft cartilage and host cartilage. The surface of the cartilage of the transplants was slightly un- 


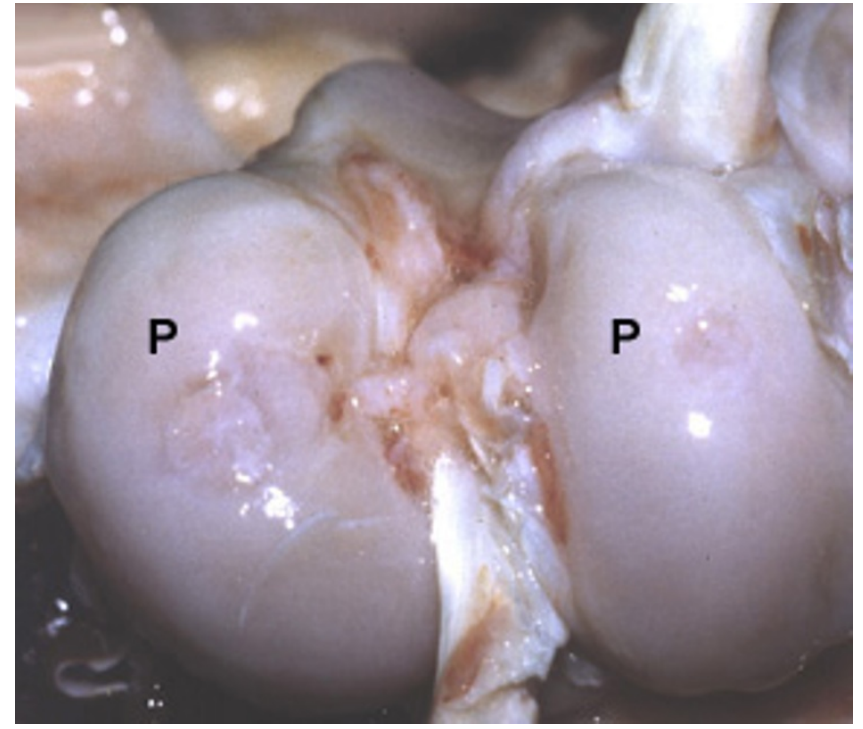

Figure 3

Macroscopic appearance of femoral condyles: photooxidized osteochondral transplants $(P)$ in both condyles after 12 months.

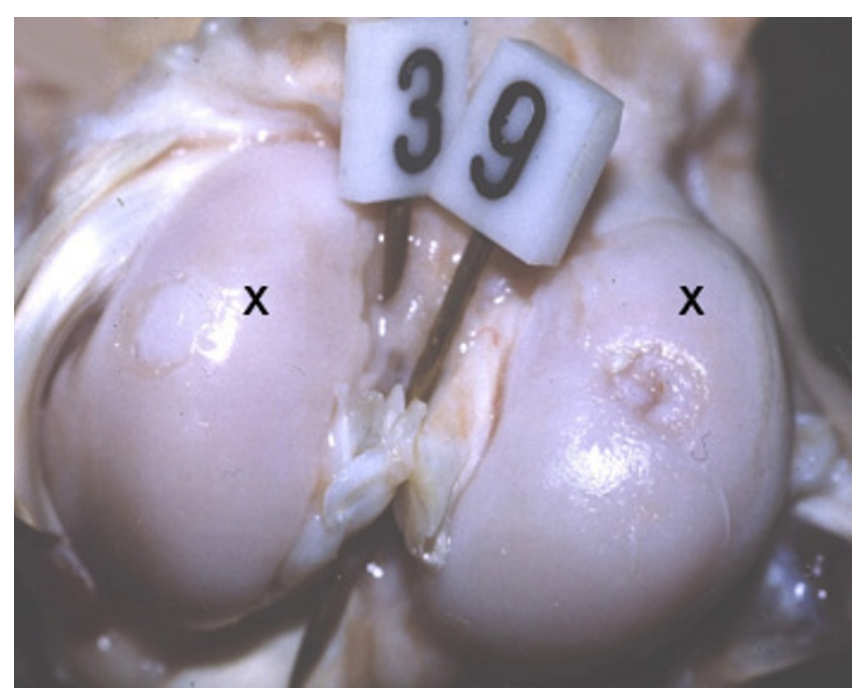

\section{Figure 4}

Macroscopic appearance of femoral condyles: xenogeneic osteochondral transplants $(X)$ in both condyles after 12 months.

even and only one transplant was surrounded by dull host cartilage. The autogeneic osteochondral transplants $(n=2)$ stayed in place, showed a slightly uneven surface and cartilage flakes at the interfaces. Interfaces between graft and host cartilage were still visible in three xenogeneic osteochondral grafts $(n=4)$ and there graft surface appeared uneven.

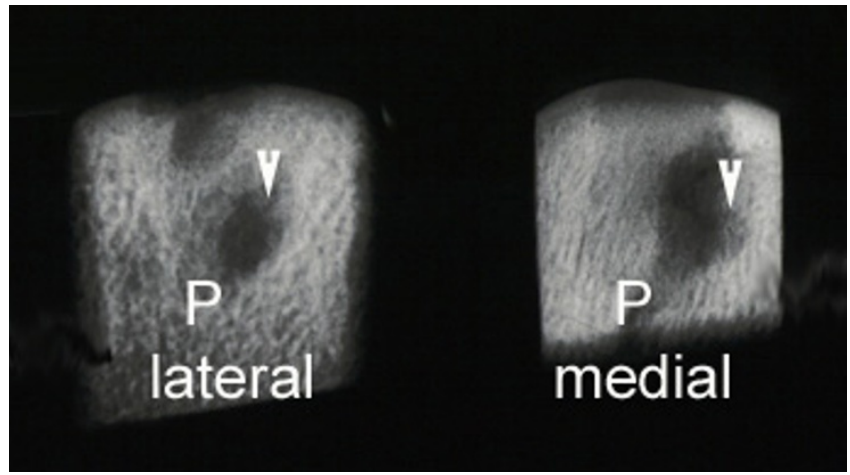

Figure 5

Microradiographs of photo-oxidized grafts in femoral condyles after 6 months (5) and 18 months (6). After 6 months cyst formation is visible (5) whereas the previously graft area cannot be seen after 18 months.

\section{Microradiography}

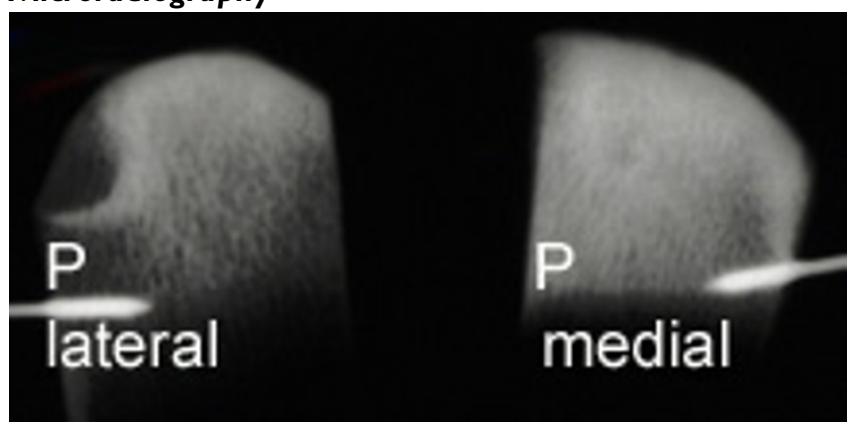

\section{Figure 6}

Microradiographs of photo-oxidized grafts in femoral condyles after 6 months (5) and 18 months (6). After 6 months cyst formation is visible (5) whereas the previously graft area cannot be seen after 18 months.

At six months in photo-oxidized (Figure 5), autogeneic and xenogeneic transplants showed radiological changes from slight inhomogenous radiolucency to severe cyst like lesions. No signs of cyst like lesions were seen at twelve months in six of 10 photo-oxidized osteochondral transplants. In the autogeneic osteochondral transplant a slight uneven subchondral contour and slight radiolucency were seen, whereas two xenogeneic osteochondral transplants showed moderate cyst like lesions. The radiological results of the osteochondral transplants at eighteen months were comparable in most cases with the twelve months results, except that in one case of the photo-oxidized transplants and in one case of the xenogeneic transplants a severe cyst like lesion at the graft-host interface was found. A microradiograph of a photo-oxidized transplant at eighteen months is shown in figure 6. 


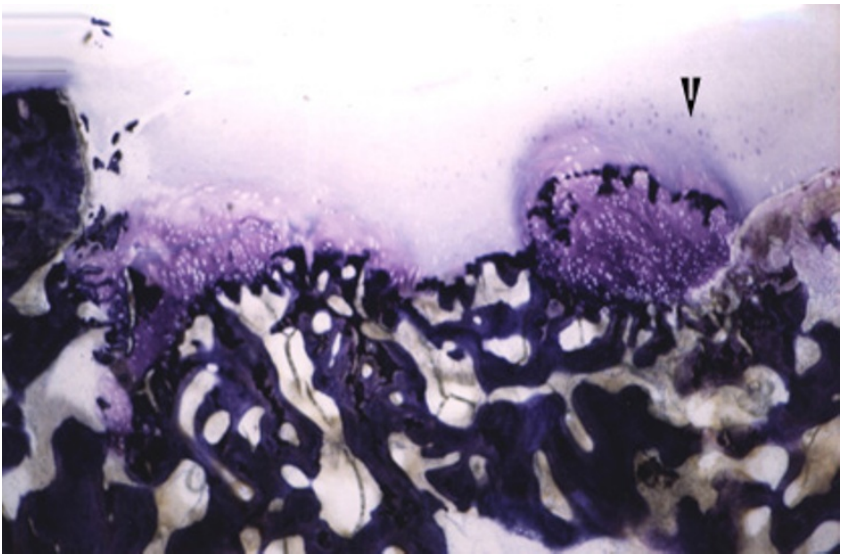

Figure 7

Histological section (toluidine blue staining; $10 \times$ ) of the femoral condyle with a photo-oxidized graft after 6 months. Arrows indicate the border between graft and host tissue and the formation of new hyaline - like tissue starting from the tide line.

\section{Histology}

6 - months group

All photo-oxidized transplants showed cystic lesions of different sizes. The border between graft and host cartilage was clearly visible in all samples and the graft cartilage showed a decreased metachromasia compared to the host cartilage. The changes in the host cartilage such as, metachromasia, cell density and fibrillation varied between samples. Increased remodelling activity of the bony part in the transplant was seen at six months compared to two months (unpublished data). At six-month first signs of cellularity occurs in 2 of 10 photo-oxidized grafts. Cells from the subchondral bone area had penetrated the calcified cartilage and differentiated into chondrocyte - like cells, arranged in clusters, nests and/ or columns (Figure 7,8). The autogeneic osteochondral transplants showed cystic lesions, separation between graft and host cartilage and an increased bone remodelling activity in the graft. The cleft between graft and host cartilage was more pronounced in the xenogeneic osteochondral transplants compared to photo-oxidized and autogeneic transplants. Cystic lesions were visible in all sections and the bony part was being remodelled.

\section{2 - months group}

In six of the photo-oxidized transplants no cystic lesions were seen. These six grafts showed metachromatic staining of the graft similar to that of the host cartilage, and a fusion between graft and host tissue. The graft cartilage appeared hyaline - like. Around the new tissue chondrocyte clusters were found. In the autogeneic osteochondral transplants cystic lesions were still visible and the host and graft cartilage remained separate. In all four xe-

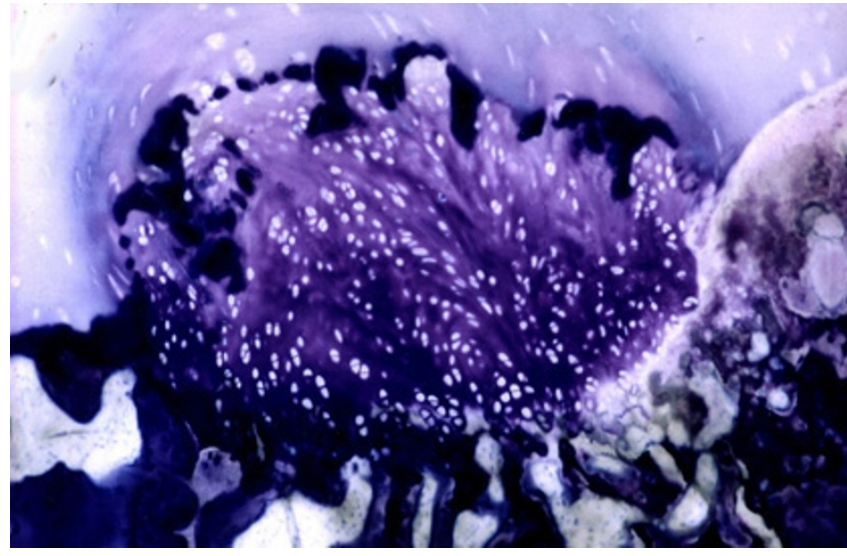

Figure 8

Close up of 7: The new tissue shows a more intensive staining compared to the original cartilage of the photo-oxidized transplant $(63 \times)$.

nogeneic grafts cystic lesions and clefts between host and graft cartilage were still seen. Cell density and metachromasia had decreased in the adjacent host cartilage of these grafts.

\section{8 - months group}

In six of ten photo-oxidized samples no cystic lesions were seen and the overall results were comparable with the twelve months results. In contrast to the photo-oxidized osteochondral grafts (Figure 9,10,11) no signs of fusion between host and graft cartilage were seen in autogeneic grafts (Figure 12, 13). The xenogeneic grafts (Figure 14, 15) still showed cystic lesions, no fusion between host and graft cartilage and severe changes of cartilage degradation such as decreased metachromasia and hypocellularity were found in the host cartilage.

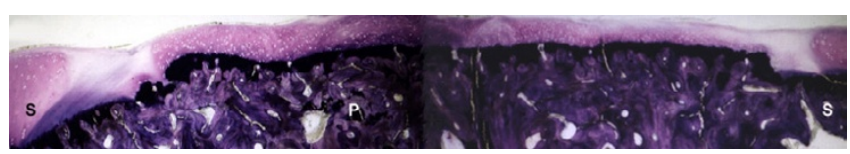

Figure 9

Histological section (toluidine blue staining; $10 \times$ ) of the femoral condyle with a photo-oxidized graft $(P)$ after 18 months. Host-graft interface is filled with low cell density tissue. (S: host bone and cartilage).

\section{Statistical analysis}

Statistical analysis was performed between photo-oxidized grafts at different time point. No analysis of autoand xenografts was performed because of the low numbers. Significantly higher scores were found in the general appearance of the graft and host $(p<0.01)$ and cartilage $(\mathrm{p}<0.05)$ in photo-oxidized grafts at 12 months 


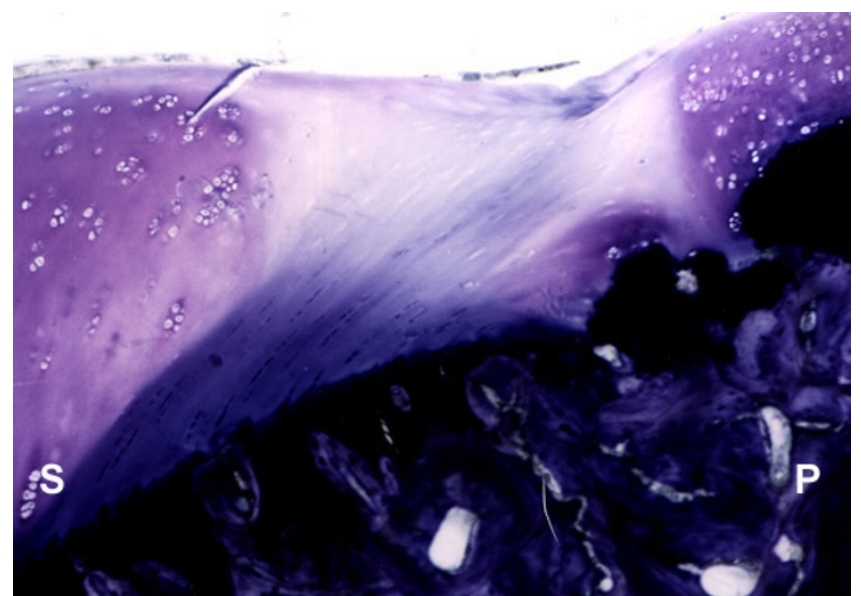

Figure 10

Close ups of $A$. The staining in the binding area is less intensive compared to the staining of the host $(S)$ and graft tissue $(\mathrm{P})$. The tide line has been completely remodelled. $(25 \times)$

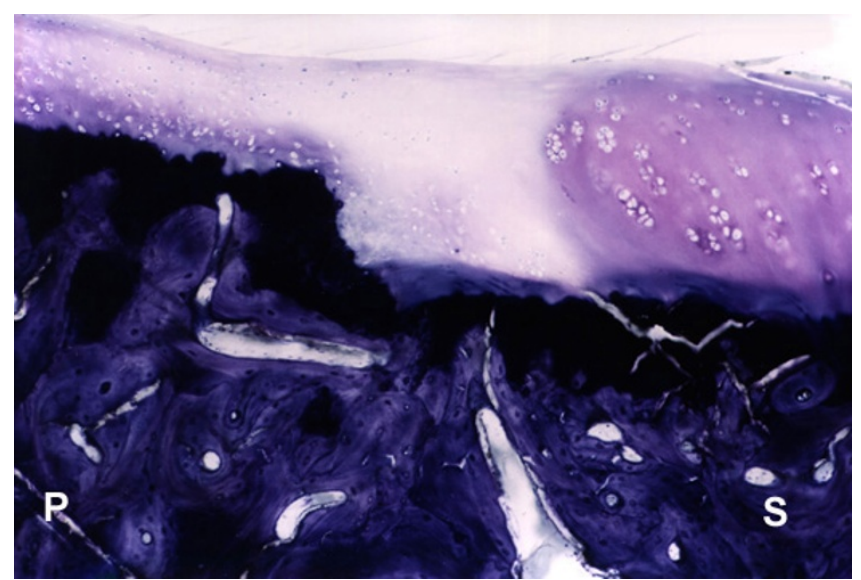

Figure I I

Close ups of $A$. The staining in the binding area is less intensive compared to the staining of the host $(S)$ and graft tissue (P). The tide line has been completely remodelled. $(25 \times)$

compared to 6 months. The general appearance included the overall impression of the graft and host at a low magnification. Cyst formation throughout the graft and host to complete remodelling of the graft and fusion of host and graft cartilage were taken into account. The appearance of the mineralised cartilage was significantly higher at 18 months in contrast to 6 months. Parameters included in the scoring were the contours, continuity, thickness of mineralised cartilage, staining and resorption of graft and host mineralised cartilage. The subchondral bone showed higher scores at $12(\mathrm{p}<0.001)$ as well as at 18 months $(\mathrm{p}<0.01)$ in comparison to 6 months. Criteria for evaluation of the subchondral bone among others

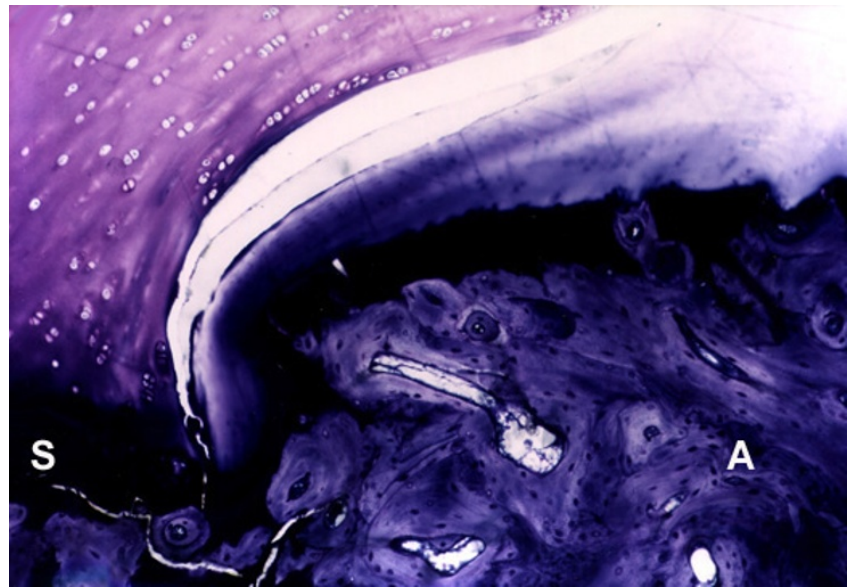

Figure 12

Histological section (toluidine blue staining; $25 \times$ ) of a medial femoral condyle with an autogeneic graft (A) after 18 months. No binding between host (S) and graft cartilage can be seen.

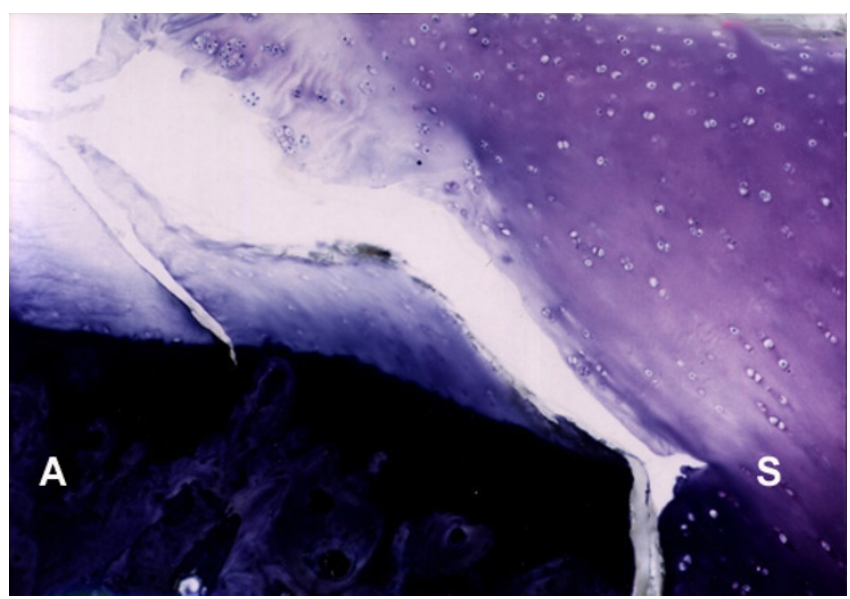

Figure 13

Histological section (toluidine blue staining; $25 \times$ ) of a medial femoral condyle with an autogeneic graft (A) after 18 months. No binding between host $(S)$ and graft cartilage can be seen. Signs of degradation in graft cartilage (A) and cluster formation ( 4 ) in host cartilage $(S)$ are visible.

were cysts formation, resorption, apposition, regularity and support of the cartilage (Table 1; Figure 1). Cyst formation was more prominent at six months compared to twelve and eighteen months, which resulted in lower scores for general appearance and subchondral bone. Fusion of graft and host cartilage was found at twelve and eighteen months, but not at six months. This leads to higher scores for cartilage at twelve and eighteen months. 


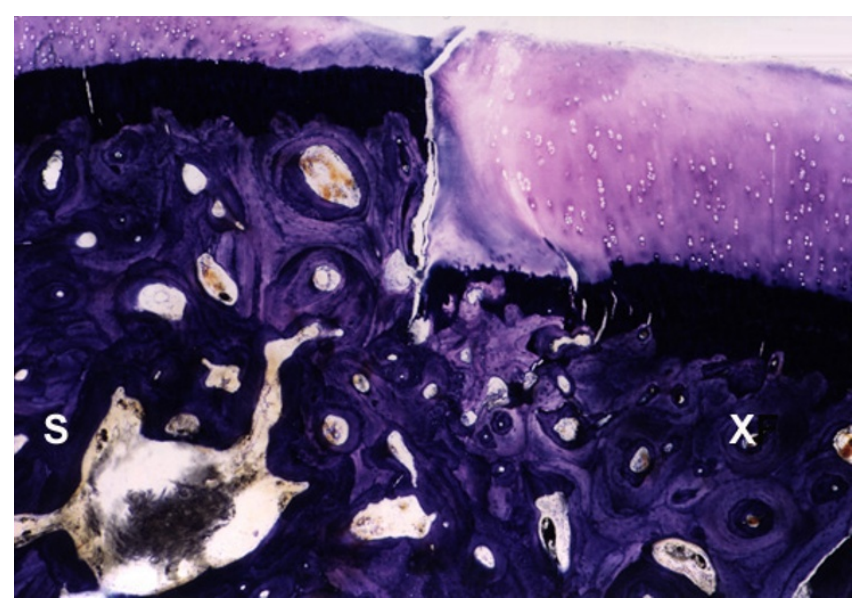

Figure 14

Histological section (toluidine blue; $25 \times$ ) of the medial femoral condyle with a xenogeneic graft $(X)$ after 14 months. No binding between host (S) and graft cartilage can be seen. Severe signs of degradation in host cartilage (S) and at the edge of the graft cartilage $(X)$ are visible.

\section{Discussion}

An osteochondral transplant pre-treated with photo-oxidation was studied in-vivo. The grafts were transplanted into the weight bearing area of the stifle joint in sheep and showed a hyaline-like cartilage matrix twelve and eighteen month after surgery. A bond at the articular surface between the host and graft was formed if joint congruity was maintained throughout the experimental period. In contrast to these findings, the control groups with auto- and untreated xenografts showed cartilage degradation and/or major gaps at the interface at eighteen months after surgery. As far as the authors are aware, this is the first time that the hyaline-like cartilage structure preservation has been demonstrated histologically at eighteen months after an osteochondral grafting procedure.

Osteochondral grafts for cartilage transplantation are effective clinically and it was suggested that they provide a better restoration of articular surfaces than the natural repair response [37].

Although the use of the pressfit-technique in the surgical procedure was proved to be suitable for this grafting method, some aspects need to be improved. It was not always possible to align the drill bit at $90^{\circ}$ to the articular surface because of the convexity of the surface. As a result the graft was introduced at a slightly oblique angle to the articular surface, leading to small steps at the interface of host and graft articular surfaces. These small steps seem to have an influence on the graft remodelling, as there may be a gap in and continuity between joint

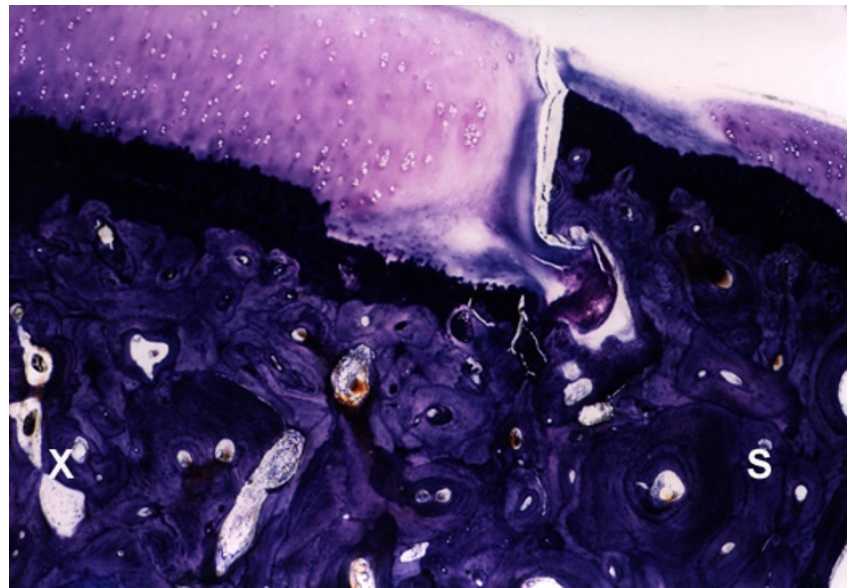

Figure 15

Histological section (toluidine blue; $25 \times$ ) of the medial femoral condyle with a xenogeneic graft $(X)$ after 14 months. No binding between host (S) and graft cartilage can be seen. Severe signs of degradation in host cartilage $(\mathrm{S})$ and at the edge of the graft cartilage $(X)$ are visible.

space and subchondral bone [38]. This continuity allows synovial fluid to penetrate the bone and may enhance cyst formation. Furthermore, attention should be placed on the thickness of the graft cartilage, which should be similar to the thickness of the ovine articular cartilage at the femoral condyle. An equal height in cartilage thickness avoids steps between the host and graft tidemark. Another problem was caused by the fact that the grafts, with the exception of the autograft, were not harvested from orthotopic areas and therefore joint congruency could not always be achieved. This is also true for techniques applied in human patients where the autografts are harvested from non-weightbearing areas within the same joint and implanted into the defect [25]. The preparation of photo-oxidized grafts in advance and a selection of a graft with an optimal fitting surface could reduce this problem.

This study demonstrated very clearly, that a final conclusion on graft survival and/or regeneration might be drawn only after an adequate length of evaluation time. Autografts looked promising at two months (preliminary study; data not shown) and even six months after surgery, but at twelve and eighteen months the cartilaginous region of the grafts had deteriorated and no living chondrocytes were present. In contrast to the autografts in this study, photo-oxidized grafts demonstrated signs of viable matrix at twelve and eighteen months after transplantation. The metachromasia of the graft cartilage matrix was increased compared to grafts at six months. When comparing these findings to literature [11] methodological differences have to be considered. In 
the present study 4.4-mm diameter autografts were used in contrast to Hurtig et al. [11], whose used 10-mm diameter autografts, which appeared indistinguishable from fresh tissue at 12 months.

The location of the grafts at the maximal weight bearing area was selected to test the grafts under maximal mechanical loads. The weight bearing area mainly requires replacement if there is localised cartilage damage. If grafts hold up to these loads, they should survive in less exposed cartilage areas as well.

The preparation of photo-oxidized grafts proved to be reliable and because they were harvested from fresh cadavers, sufficient numbers was not a problem. The sterilisation procedure for the grafts, which allows storage over a longer period of time, has to be optimised and quality assurance provided. Both procedures are under current investigation.

Although a scoring system was developed to assess graft performance objectively, caution is indicated in judging statistical results. Despite the fact that some grafts had good scores for matrix viability and/or subchondral bone remodelling, the overall performance of the graft may still be hampered by the absence of cartilage bonding between graft and host tissues. It is difficult to know which feature of graft integration is most important for the clinical performance in the joint of a future patient. The overall assessment has to include cartilage matrix structure, stage of degradation, subchondral bone density, reconstruction of calcified cartilage and tidemark in the graft and probably most importantly the cartilage interface between graft and host. The cartilage of the photooxidized grafts at twelve and eighteen months appeared white, the original blue colour had disappeared. This result could be a sign of complete restored graft cartilage. The fact that photo-oxidized osteochondral grafts showed a hyaline-like cartilage matrix with apparently living and matrix synthesising chondrocytes eighteen months after transplantation is unique and has never been reported. Furthermore, fusion of host and graft interface after that time period was not observed in autografts and untreated xenografts [9].

Cyst-like lesions developed in all types of grafts. However, if joint congruity was maintained throughout the study, they were usually less pronounced in the photooxidized grafts than in the auto- and xenografts. In these cases the bony part of the graft was resorbed more slowly compared to the controls and bone remodelling followed the classical principle of creeping substitution [39]. It seems that the structure of the bone of the photo-oxidized grafts was more stable. This may be a result of the photo-oxidation process, which may change the immu- nologic response of the host bone [40,41] and also increase the linkage of collagen fibers in the bone matrix and so affect its structural properties. In this study, autografts and untreated xenografts were used as control to investigate the influence of photo-oxidation. In a later study, untreated and photo-oxidized allografts were added as controls to compare these to already used controls and photo-oxidized xenografts (not published yet).

It appeared that the process of photo-oxidation influenced the response of the adjacent tissue, possibly as a result of the diffusion of the methylene blue solution into the host cartilage. This diffusion was observed one day after an in-vitro implantation test when a photo-oxidized graft was implanted in a femoral condyle. The methylene blue solution, remaining after photo-oxidation, may inhibit adjacent matrix degeneration while at the same time maintaining matrix synthesis. This interaction may influence the growing of tissue between host and photo-oxidized grafts in contrast to auto- and xenografts. The degeneration of the host matrix has been observed in other studies [20,28,37,42], but the importance of this feature was not established.

The matrix of the photo-oxidized transplants showed at the time point of surgery a decrease in metachromatic staining. Furthermore, in the in-vitro study of the photooxidized cartilage it was shown, using the live/dead staining, that no viable chondrocytes are present after the process of photo-oxidation [33]. Chondrocyte clusters were found within the photo-oxidized graft at six months and fusion between host and graft cartilage of the photo-oxidized grafts were seen at twelve and eighteen months. The evidence of chondrocyte clusters is a sign of intrinsic repair, but in these grafts the source of the cells seem to be extrinsic. Extrinsic repair is caused by cells from outside to the cartilage [4]. Since the result of the grafts is good, it seems that the importance of chondrocyte viability at the time of transplantation may not be a crucial point for the outcome of the grafts $[9,23]$. It may be more important to provide an optimal structure for cartilage resurfacing where mesenchymal cells from the subchondral bone area may grow and differentiate into matrix synthesising chondroblasts and chondrocytes. The advantage of preparing osteochondral transplants in advance over collecting autografts within another joint of the same individual at the time of surgery is obvious and needs no further explanation [8$12,20]$. However, the fate of xenografts pre-treated with photo-oxidation has to be investigated further. Crucial questions to be answered before pre-treated xenografts may be used in human patients are whether these grafts are incorporated or whether they are replaced. Also the immunologic response of the host tissue needs further attention, although the present study indicated that the 
process of photo-oxidation seems to inhibit the antigenic response of the host tissue to the grafts, as it was reported for photo-oxidized pericardial tissue [31,40,41,43]. Allogeneic osteochondral grafts were cryopreserved, that is to reduce the immunological response and prepare the graft in advance. In contrast to the present study, autografts showed better results than the cryopreserved grafts [23]. The approach of graft preparation may have an influence on the interaction of the graft with host tissue and its outcome.

The present study showed that bovine xenografts, pretreated with photo-oxidation, are suitable osteochondral grafts for cartilage replacement in sheep. The appearance of a hyaline-like cartilage with matrix producing chondrocytes eighteen months after transplantation is promising and deserves further investigation. Future investigations will focus on optimising the shape of the grafts and the achievement of joint congruity including a water tight seal at the level of the calcified cartilage. Studying apoptotic activity of the host and graft chondrocytes may help to define the nature of the grafts and its ultimate fate. Further immunology studies should include tests for identifying the origin of cells in the graft.

\section{Conclusions}

The pre-treatment of osteochondral grafts with the process of photo-oxidation appears to have a beneficial effect on cartilage and bone remodelling. The use of photo-oxidized osteochondral transplant may be considered for articular cartilage replacement and therefore may delay artificial joint replacements in human patients.

\section{Competing interests}

The research project was funded by Sulzer Orthopedics. It is not known whether they will gain financially from the publication of this paper.

\section{Acknowledgements}

Sulzer Orthopedics Ltd., Winterthur, Switzerland, provided support for this work. The authors thank Prof. U. Hübscher, Institute of Biochemistry, Zürich, and Dr. D. Ashhurst, London, for reviewing the manuscript.

\section{References}

I. Mankin HJ: Biology of Autologous Cartilage Repair. In: steochondral Allografts Edited by Friedlaender GE, Sell KW, Mankin HJ. pp. 59-72. Boston/Toronto: Little, Brown and Company; 198359-72

2. Caplan Al, Elyaderani M, Mochizuki Y, Wakitani S, Goldberg VM: Principles of cartilage repair and regeneration. Clin, Orthop, 1997254-269

3. Hunziker EB, Rosenberg L: Articular Cartilage Repair. In: Arthritis and Allied Conditions Edited by Koopmann WJ, vol. 2, 13 ed. pp. 20272938. Baltimore, MA: Williams and Wilkins; 19972027-2938

4. Hurtig MB, Fretz PB, Doige CE, Schnurr DL: Effects of lesion size and location on equine articular cartilage repair. Can. J. Vet. Res 1988, 52:137-146

5. Hanie EA, Sullins KE, Powers BE, Nelson PR: Healing of full-thickness cartilage compared with full-thickness cartilage and subchondral bone defects in the equine third carpal bone. Equine Vet. J 1992, 24:382-386
6. Convery FR, Akeson $\mathrm{WH}$, Keown GH: The repair of large osteochondral defects. An experimental study in horses. Clin. Orthop. Relat. R 1972, 82:253-262

7. Weisbrode SE, Doige CE: Bone and Joints. In: Special Veterinary Pathology Edited by McGavin MD, Carlton WW, Zachary JF, 3 ed. pp. 49952 I. St. Louis - London: Mosby; 200I499-52I

8. Mow VC, Ratcliffe A, Rosenwasser MP, Buckwalter JA: Experimental studies on repair of large osteochondral defects at a high weight bearing area of the knee joint: a tissue engineering study. J. Biomech. Eng 1991, I I 3:198-207

9. Shahgaldi BF, Amis AA, Heatley FW, McDowell J, Bentley G: Repair of cartilage lesions using biological implants. A comparative histological and biomechanical study in goats. J. Bone Joint Surg. $\mathrm{Br}$ 1991, 73:57-64

10. Brittberg M, Lindahl A, Nilsson A, Ohlsson C, Isaksson O, Peterson $\mathrm{L}$ : Treatment of deep cartilage defects in the knee with autologous chondrocyte transplantation [see comments]. $\mathrm{N}$. Engl. J. Med 1994, 331:889-895

11. Hurtig MB, Novak K, McPherson R, McFadden S, McGann LE, Mul drew K, Schachar NS: Osteochondral dowel transplantation for repair of focal defects in the knee: an outcome study using an ovine model. Vet. Surg 1998, 27:5-16

12. Wohl G, Goplen G, Ford J, Novak K, Hurtig M, McPherson R, McGann L, Schachar N, Zernicke RF: Mechanical integrity of subchondral bone in osteochondral autografts and allografts. Can. J. Surg 1998, 4I:228-233

13. Desjardins MR, Hurtig MB, Palmer NC: Incorporation of fresh and cryopreserved bone in osteochondral autografts in the horse. Vet. Surg 1991, 20:446-452

14. Garrett JC: Osteochondral allografts. Instr. Course Lect 1993, 42:355-358

15. Garrett JC: Fresh osteochondral allografts for treatment of articular defects in osteochondritis dissecans of the lateral femoral condyle in adults. Clin. Orthop. Relat. R 199433-37

16. Zarnett R, Salter RB: Periosteal neochondrogenesis for biologically resurfacing joints: its cellular origin. Can. J. Surg 1989, 32:17I-174

17. Kreder HJ, Moran M, Keeley FW, Salter RB: Biologic resurfacing of a major joint defect with cryopreserved allogeneic periosteum under the influence of continuous passive motion in a rabbit model. Clin. Orthop. Relat. $R$ 1994288-296

18. Hendrickson DA, Nixon AJ, Grande DA, Todhunter RJ, Minor RM, Erb H, Lust G: Chondrocyte-fibrin matrix transplants for resurfacing extensive articular cartilage defects. J. Orthopaed. Res 1994, I 2:485-497

19. Grande DA, Halberstadt C, Naughton G, Schwartz R, Manji R: Evaluation of matrix scaffolds for tissue engineering of articular cartilage grafts. J. Biomed. Mater. Res 1997, 34:2 II-220

20. Gilbert JE: Current treatment options for the restoration of articular cartilage. Am. J. Knee Surg 1998, I I:42-46

21. Outerbridge HK, Outerbridge AR, Outerbridge RE: The use of a lateral patellar autologous graft for the repair of a large osteochondral defect in the knee. J. Bone Joint Surg. Am 1 995, 77:6572

22. Buckwalter JA, Mankin HJ: Articular cartilage: tissue design and chondrocyte-matrix interactions. Instr. Course Lect 1998, 47:477486

23. Schachar NS, Novak K, Hurtig M, Muldrew K, McPherson R, Wohl G, Zernicke RF, McGann LE: Transplantation of cryopreserved osteochondral Dowel allografts for repair of focal articular defects in an ovine model. J. Orthop. Res 1999, 17:909-919

24. Laprell H, Petersen W: Autologous osteochondral transplantation using the diamond bone-cutting system (DBCS): 6-12 years' follow-up of 35 patients with osteochondral defects at the knee joint. Arch Orthop Trauma Surg 200 I, I 2 I:248-253

25. Hangody L, Kish G, Karpati Z, Szerb I, Udvarhelyi I: Arthroscopic autogenous osteochondral mosaicplasty for the treatment of femoral condylar articular defects. A preliminary report. Knee Surgery, Sports Traumatology, Arthroscopy 1997, 5:262-267

26. Schachar N, McAllister D, Stevenson M, Novak K, McGann L: Metabolic and biochemical status of articular cartilage following cryopreservation and transplantation: a rabbit model. J. Orthop. Res 1992, 10:603-609

27. Malinin TI, Mnaymneh W, Lo HK, Hinkle DK: Cryopreservation of articular cartilage. Ultrastructural observations and long- 
term results of experimental distal femoral transplantation. Clin. Orthop. Relat. R 199418-32

28. Shapiro F, Koide S, Glimcher MJ: Cell origin and differentiation in the repair of full-thickness defects of articular cartilage. J. Bone Joint Surg. Am 1993, 75:532-553

29. Sullins KE, Veit HP, Mcllwraith CW: Osteochondral grafts to fill large articular defects in horses. Vet. Surg 1989, 1 8:77

30. Rodrigo JJ: The Problem of Fit in Osteocartilaginous Allografts. In: Osteochondral Allografts Edited by Friedlaender GE, Sell KW, Mankin HJ. pp. 249-255. Boston/Toronto: Little, Brown and Company; 1983249-255

31. Moore MA, Bohachevsky IK, Cheung DT, Boyan BD, Chen WM, Bickers RR, Mcllroy BK: Stabilization of pericardial tissue by dyemediated photooxidation. J. Biomed. Mater. Res I994, 28:6 I I-6I8

32. Mcllroy BK, Robinson MD, Chen WM, Moore MA: Chemical modification of bovine tissues by dye-mediated photooxidation. J. Heart Valve Dis 1997, 6:416-423

33. Akens MK, von Rechenberg B, Bittmann P, Nadler D, Zlinszky K, Auer JA: In-vitro Studies of a Photo-oxidized Bovine Articular Cartilage. Journal of Veterinary Medicine A 200 I

34. Birkedal-Hansen $\mathrm{H}$ : Role of cytokines and inflammatory mediators in tissue destruction. J. Periodontal. Res 1993, 28:500-510

35. Birkedal-Hansen H, Moore WG, Bodden MK, Windsor LJ, BirkedalHansen B, DeCarlo A, Engler JA: Matrix metalloproteinases: a review. Crit. Rev. Oral Biol. M I993, 4:197-250

36. von Rechenberg B, Leutenegger C, Zlinszky K, Mcllwraith CW, Akens MK, Auer JA: Upregulation of mRNA of interleukin- $I$ and -6 in subchondral cystic lesions of four horses. Equine Vet. J 200I, 33:143-149

37. Buckwalter JA, Mankin HJ: Articular cartilage: degeneration and osteoarthritis, repair, regeneration, and transplantation. Instr. Course Lect 1998, 47:487-504

38. Frisbie DD, Trotter GW, Powers BE, Rodkey WG, Steadman JR, Howard RD, Park RD, Mcllwraith CW: Arthroscopic subchondral bone plate microfracture technique augments healing of large chondral defects in the radial carpal bone and medial femoral condyle of horses. Vet. Surg 1999, 28:242-255

39. Phemister DB, Gordon JE: The Etiology of Solitary Bone Cyst. The Journal of the American Medical Association 1926, 87:1429-1433

40. Moore MA, Phillips RE: Immunology and biocompatibility of pericardial tissue stabilized by dye-mediated photooxidation. In: The 20th Annual Meeting of the SOCIETY FOR BIOMATERIALS; 1994; Boston, MA, USA

41. Moore MA: Pericardial tissue stabilized by dye-mediated photooxidation: a review article. J. Heart Valve Dis 1997, 6:52I-526

42. Flynn JM, Springfield DS, Mankin HJ: Osteoarticular allografts to treat distal femoral osteonecrosis. Clin. Orthop. Relat. R I9943843

43. Moore MA, Phillips RE: Biocompatibility and immunologic properties of pericardial tissue stabilized by dye-mediated photooxidation. J. Heart Valve Dis 1997, 6:307-315

Publish with BioMed Central and every scientist can read your work free of charge

"BioMedcentral will be the most significant development for disseminating the results of biomedical research in our lifetime."

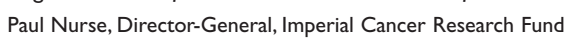

Publish with BMC and your research papers will be:

- available free of charge to the entire biomedical community

- peer reviewed and published immediately upon acceptance

- cited in PubMed and archived on PubMed Central

- yours - you keep the copyright
BioMedcentral.com editorial@biomedcentral.com 